\title{
A Vivência Interdisciplinar no Ensino Superior: Um Estudo de Projetos Pedagógicos
}

\author{
Paulo de Tarso Oliveira ${ }^{1}$ e Maria Eunice Barbosa Vidal ${ }^{2}$ \\ ${ }^{1}$ Centro Universitário Municipal de Franca - Uni-FACEF, Brasil. | ptarsoliveira@gmail.com | \\ https://orcid.org/0000-0002-5739-4827 \\ ${ }^{2}$ Universidade Federal do Triângulo Mineiro - UFTM, Brasil. | \\ mariaeunice.vidal@uftm.edu.br | https://orcid.org/0000-0002-8434-143X
}

\begin{abstract}
Resumo: Esta investigação comporta a questão básica de como a necessidade de uma vivência interdisciplinar é tratada nos projetos pedagógicos de ensino superior. A escolha se justifica pela necessidade de compreensão da interdisciplinaridade como demanda para as instituições. O objetivo geral é ampliar a compreensão do percurso institucional das proposituras gerais de interdisciplinaridade das Diretrizes Curriculares para o ensino superior. Especificamente, é descrever e interpretar aspectos das concepções de interdisciplinaridade contidas nos projetos pedagógicos dos cursos. A metodologia utilizada envolve a coleta de dados e sua interpretação. Os dados serão coletados em projetos pedagógicos disponíveis em sites de instituições universitárias situadas numa região delimitada. Para a interpretação, foram estabelecidas categorias preliminares de análise que englobam características específicas dos projetos pedagógicos, bem como as correspondências referentes às Diretrizes Curriculares, às diferenças e semelhanças entre projetos de diferentes modalidades de graduação, quanto à interdisciplinaridade. Tais categorias são: (i) correspondência em relação à respectiva Diretriz Curricular; (ii) fundamentação teórico / prática; (iii) consistência: coerência / contradição entre a proposta de interdisciplinaridade e demais concepções e proposituras do curso; (iv) previsão de grau e intensidade de envolvimento individual e institucional nas ações interdisciplinares; (v) similaridade / diferenciação das concepções de interdisciplinaridades entre projetos nas áreas de Ciências Humanas, Exatas e Tecnológicas, Biológicas; (vi) similaridade / diferenciação das concepções de interdisciplinaridade entre projetos das diferentes modalidades de graduação: bacharelado, licenciatura, formação de tecnólogos. Os resultados até o momento estão restritos à construção de um referencial teórico e ao instrumental para a análise dos dados.
\end{abstract}

Palavras-chave: Interdisciplinaridade; Pesquisa Qualitativa; Projeto Pedagógico.

Institutional Implications in the Articulation of Interdisciplinarity in Higher Education: a Study of Pedagogical Projects

\begin{abstract}
This investigation involves the basic question of how the need for an interdisciplinary experience is dealt with in higher education pedagogical projects. The choice is justified by the need to understand interdisciplinarity as a demand for institutions. The general objective is to broaden the understanding of the institutional path of the general interdisciplinarity propositions of the Curricular Guidelines until their concreteness in academic practices. Specifically, it is to describe and interpret aspects of the concepts of interdisciplinarity contained in the pedagogical projects of the courses. The methodology used involves data collection and interpretation. The data will be collected in pedagogical projects available on the websites of university institutions located in a defined region. For the interpretation, preliminary categories of analysis were established that encompass specific characteristics of the pedagogical projects, as well as the correspondences referring to the Curricular Guidelines, to the differences and similarities between projects of different graduation modalities, regarding the interdisciplinarity. Such categories are: (i) correspondence in relation to the respective Curricular Guideline; (ii) theoretical / practical basis; (iii) consistency: coherence / contradiction between the interdisciplinary proposal and other conceptions and propositions of the course; (iv) forecasting the degree and intensity of individual and institutional involvement in interdisciplinary actions; ( $v$ ) similarity / differentiation of the concepts of interdisciplinarities between projects in the areas of Human Sciences, Exact and Technological, Biologica Sciences; (vi) similarity / differentiation of the concepts of interdisciplinarity between projects of different graduation modalities: bachelor's degree, licentiate degree, training of technologists. The results so far are restricted to the construction of a theoretical framework and the instrumental for the analysis of the data.
\end{abstract}

Keywords: Interdisciplinarity; Qualitative Research; Pedagogical Project. 


\section{Introdução}

Este trabalho tem origem em estudos anteriores dos autores acerca da temática da interdisciplinaridade. Em seu percurso, esses estudos - a que se somam as vivências dos autores na docência de ensino universitário - foram feitos em busca de um objetivo geral de contribuir na elaboração de indicadores e subsídios para a implantação e desenvolvimento de programas de natureza interdisciplinar, no contexto de ensino superior.

Esses desígnios permeiam a propositura da presente pesquisa, procurando dar continuidade investigativa quanto a questões e dilemas da interdisciplinaridade, tanto no plano do conhecimento quanto na consideração de sua presença na práxis do ensino superior.

O projeto, em andamento, trata da questão básica de como a interdisciplinaridade requerida nas Diretrizes Curriculares Nacionais - é entendida, no plano institucional, ou seja, como a necessidade de promover a interdisciplinaridade é tratada por instituições de ensino superior (IES), nos seus respectivos projetos pedagógicos. Fazendo um ligeiro parêntese: a denominação de Projeto Pedagógico, na legislação do ensino superior brasileiro, refere-se ao instrumento pelo qual as instituições universitárias concebem e regulam os seus cursos de graduação.

Essa questão básica se traduz em diversas questões específicas, a exemplo de: (i) a interpretação feita pela instituição acerca da interdisciplinaridade, indicada na respectiva Diretriz Curricular, traz alguma inovação ou é mera reprodução do texto?; (ii) a prática de interdisciplinaridade proposta no projeto pedagógico tem fundamentação teórica explícita?; (iii) a proposta de interdisciplinaridade é coerente com as demais concepções do projeto?; (iv) qual a previsão de participação de pessoas e da instituição na prática da interdisciplinaridade?; (v) existem diferenças significativas - na concepção de interdisciplinaridade - entre os projetos pedagógicos de cursos de diversas áreas do Conhecimento (Ciências Humanas, Exatas, Biológicas), como também entre os de diversas modalidades de curso (Bacharelado, Licenciatura, Formação de Tecnólogos)?

Em termos metodológicos, esboça um estudo documental por meio de informações acerca da interdisciplinaridade, extraídas de projetos pedagógicos de cursos de graduação contidos nos sites de IES.

\section{Referencial Teórico}

A fundamentação teórica, até agora construída, contemplou alguns aspectos históricos e conceituais, mas continua sendo desenvolvida para aprofundamento teórico e metodológico. Para tanto estão sendo considerados autores como Falcus et al. (2019), Neves (2019), Barreto et al. (2016), Silva (2019), Santos et al. (2019), entre outros. Historicamente, um ponto parece pacífico: no final do século XX e início do século XXI, a palavra interdisciplinaridade torna-se presente e proeminente no mundo educacional. Fazenda (2012, p. 42) assinalou em um texto que escreveu na década de 90: "A palavra de ordem desse final de século é interdisciplinaridade na educação".

$\mathrm{Na}$ linha do tempo, cumpre notar que, no Brasil, a partir da Lei de Diretrizes e Bases da Educação Nacional, de 1995, são emanadas pelo Conselho Nacional de Educação, as Diretrizes Curriculares dos cursos superiores. Incluiu a abordagem interdisciplinar como indicador para os projetos pedagógicos dos cursos de graduação.

Como se percebe, o termo passa a ser um dos mais representativos de aspirações educacionais no âmbito da produção do conhecimento, como forma de superação do paradigma cartesiano - de separação rígida entre sujeito e objeto -; e da superação do conhecimento produzido e transmitido em compartimentos estanques. 
Tal demanda implica em um reconhecimento da multidimensionalidade e da diversidade dos fenômenos e, igualmente, da interdependência dos objetos, fazendo com que a dinâmica de produção do conhecimento necessite de uma forte ligação entre as disciplinas e, muitas vezes, de uma aproximação às demandas sociais (Pena Vega, 2010).

$O$ autor prossegue assinalando a necessidade de comunicação efetiva entre os diversos mundos vividos pelo homem. Assim, entende que a interdisciplinaridade reside em nosso modo de ver a realidade, através do conhecimento, e na forma de lidar com essa realidade.

Em razão da complexidade cada vez maior dos fenômenos, surge a necessidade de produções do conhecimento fora das fronteiras de cada disciplina. A solução de vários problemas pode ser invisível no seio de uma única disciplina. Ainda, na produção do conhecimento, um fenômeno se desvela ao mesmo tempo que se vela: há um continuum de surgimento de novas questões a partir de cada descoberta, de cada nova explicação. Isso torna forçoso reconhecer que a apreensão da realidade é incompleta no âmbito de cada campo disciplinar.

Questões dessa natureza reforçam as preocupações em melhor compreender as relações entre disciplinas. Conceitos como multidisciplinaridade, pluridisciplinaridade, interdisciplinaridade, transdisciplinaridade, vêm a representar a necessidade de estabelecer e explicar pontes entre disciplinas (Basarab Nicolescu, 2010).

Nesse sentido, destaca-se uma evolução acentuada dos estudos sobre a interdisciplinaridade que se multiplicam e diversificam a partir da segunda metade do século 20, configurando, na atualidade, um panorama bastante amplo. A propósito, Fazenda et al. (2015) mencionam quatro direções atualmente identificáveis da pesquisa sobre interdisciplinaridade: (i) interdisciplinaridade profissional; (ii) interdisciplinaridade científica; (iii) interdisciplinaridade prática; (iv) interdisciplinaridade metodológica.

Em acréscimo, há que se mencionar que a crescente complexidade - do conhecimento e da vida atual - acelerada pela quantidade, intensidade e variedade da produção de ciência e tecnologia engendrou novas demandas sociais, especialmente, na formação profissional. Roggero (2010) comenta que, no plano da formação profissional, as exigências de novas competências socioprofissionais implicam igualmente em novas abordagens de organização e funcionamento do ensino.

A autora, estudando tais competências, propõe o seu agrupamento em quatro categorias: (i) redirecionamento do pensamento lógico-abstrato: implica na visão do generalista além do especialista e no entendimento da criatividade como habilidade para solução de problemas e não como talento inato; (ii) Comunicação e linguagens do mundo contemporâneo: implica nas habilidades de situar-se nas redes de informação, o conhecimento de outras línguas além de aperfeiçoamento do domínio da língua materna e compreensão da linguagem não verbal; (iii) Trabalho em equipes multidisciplinares: implica em novos tipos de relações socioprofissionais, com intercâmbios de informações e conhecimentos interdisciplinares, bem como o desenvolvimento de uma liderança educadora, para além das habilidades de mando; (iv) Capacidade de adaptação à mudança: implica no desenvolvimento de pensamento estratégico, com habilidades de identificação de tendências e foco nas questões sociais.

Todas essas considerações evidenciam a interdisciplinaridade como marca necessária na atividade educacional. Nesse sentido, Feldman (2014) observa que o ideal de uma formação interdisciplinar é parte integrante das políticas públicas atinentes ao ensino superior brasileiro, cujas orientações e normas balizam as concepções e ações educativas das instituições, consubstanciadas em seus respectivos projetos pedagógicos. 


\subsection{A Interdisciplinaridade como Pretensão Educacional}

A interdisciplinaridade é vista como uma espécie de devir: algo desejável na atitude do homem em buscar a compreensão da realidade, enfim, do entendimento de seu mundo. Japiassu (1992) reforçou essa ideia de interdisciplinaridade como atitude quando intitulou artigo para a Revista Tempo Brasileiro, como $A$ atitude interdisciplinar no sistema de ensino.

Jantsch e Bianchetti (2011) assinalaram a validade dessa pretensão, uma vez que em muitas temáticas se impõe o princípio da interdisciplinaridade. A interdisciplinaridade decorre de esforços na construção do conhecimento e seu ensino. Para sua plenificação, há implicações pessoais e institucionais a considerar.

Em obra clássica sobre a interdisciplinaridade, Santomé (1998) propõe quatro categorias que as englobam:

- Intencionalidade: fazer dialogar o que se isolou e; até mesmo, em certos casos, de reunificar o que se fragmentou.

- Mudanças nas estruturas institucionais: promover a ligação mais estreita entre as instituições universitárias com as demais esferas da sociedade.

- Mudança da vida acadêmico-universitária: tornar a universidade um amplo laboratório de conhecimento / pensamento, com a criação de modelos mais explicativos desta realidade tão complexa e difícil de abranger

- Prática pedagógica: construir novas estruturas mentais, novos conteúdos e novas metodologias.

Depreende-se o papel proeminente atribuído às instituições na busca de implementação da marca interdisciplinar na abordagem do conhecimento. Já no início deste século, Coimbra (2000) alertava para a necessidade de uma vocação interdisciplinar que parece ser muito mais das instituições do que de indivíduos isolados. Ainda que existam esforços individuais bem sucedidos de superação de uma formação disciplinar para níveis interdisciplinares; tal transformação, na maioria das vezes, precisa ser despertada, incentivada e desenvolvida pelas instituições.

\section{Metodologia}

O desenho metodológico desta pesquisa documental propõe uma etapa referente ao estudo de projetos pedagógicos acessados em sites de IES, prevendo-se análise de 15 (quinze) projetos, numa abordagem qualitativa.

\subsection{Pesquisa Documental}

Conforme já exposto, a pesquisa que ora se delineia é motivada por estudos anteriores. $\mathrm{O}$ primeiro deles consistiu em investigação junto a professores de ensino superior e o segundo, com coordenadores de cursos de graduação em IES. Em ambos, os resultados evidenciaram a dicotomia discurso / prática, quanto à interdisciplinaridade no exercício da docência. Mostraram, no primeiro estudo, uma contradição, no plano das atitudes docentes, caracterizadas pela exteriorização de um discurso que enaltecia e cultuava a interdisciplinaridade (como forma de abordagem da realidade e como necessidade nas lides do ensino); e, por outro lado, as raras e quase inexistentes práticas de promoção da interdisciplinaridade na docência no ensino superior.

O segundo estudo levantou pontos que apoiavam e os que dificultavam a compatibilidade entre o discurso e a prática da interdisciplinaridade nas atividades do ensino superior. Foram apontados aspectos da representação que os coordenadores fazem do termo interdisciplinaridade e a forte presença da contradição discurso / prática, tanto quanto nos resultados de pesquisa com docentes. 
A intenção atual é coletar dados de instituições situadas num raio aproximado de $200 \mathrm{Km}$ em torno das cidades de Franca, no Estado de São Paulo, e da cidade de Uberaba, no Estado de Minas Gerais. Justifica-se, primeiramente, porque tais regiões comportam um grande número de IES. Outra razão é o interesse por estudos regionalizados, tendo em vista a atuação de um dos autores em Programa de Mestrado em Desenvolvimento Regional. A terceira razão é a que são cidades-sede de docência dos autores desta pesquisa.

Os projetos incluem-se como documentos. Entendemos documento como "qualquer suporte que contenha informação registrada, formando uma unidade, que possa servir para consulta, estudo ou prova" (ABNT 2018, p 2).

Justifica-se essa modalidade de pesquisa sobretudo quando consideramos, com Flick (2009), que a construção de explicações sobre eventos pode ser feita a partir de documentos entendidos não como contêineres de informação; mas, sim, como instrumentos de contextualização da informação metodologicamente elaborados.

Visto assim, cabe mencionar que, na pesquisa qualitativa, deve ficar clara a inserção do pesquisador no objeto. Tanto as suas motivações, quanto a sua capacidade de observação e compreensão; quanto, ainda, a sua visão de mundo estão presentes na sua investigação, o que leva a uma visão influenciada pelos significados que ele tem incorporados pelas suas práticas sociais.

Sob essa chave interpretativa, Bortoni-Ricardo (2013, p. 32) defende que o pesquisador "não é um relator passivo, mas um agente ativo." Esses aspectos todos carecem de ser levados em conta, tendo em vista que a metodologia concentra-se na interpretação que busca entender fenômenos humanos observados em um determinado contexto sociocultural.

Se é válida essa ótica, também é forçoso acrescentar que a análise interpretativa documental - metodologia que responde bem à questão desta investigação - às vezes é permeada por dados observacionais, obtidos pelos autores em sua vivência profissional, incluindo a participação na elaboração de projeto pedagógico. Todos convergentes para a verificação das propostas de construção interdisciplinar nos projetos pedagógicos de cursos.

Um estudo inicial foi constituído de levantamento e análise preliminar de quatro projetos pedagógicos (um de Curso de Enfermagem, um de Curso de Direito, um de Curso de Engenharia e um de Curso de Licenciatura). Resultou daí a proposta de categorias de coleta e análise de conteúdo dos projetos pedagógicos.

\subsection{O Corpus}

Considerando todos os aspectos supramencionados, prevê-se o corpus a ser constituído por um total, já mencionado em parágrafo anterior, de 15 (quinze) projetos pedagógicos, sendo 5 (cinco) da área de Ciências da Saúde, 5 (cinco) da área de Ciências Exatas e Tecnológicas e 5 (cinco) da área de Ciências Humanas, contemplando-se, entre eles, projetos de licenciatura, bacharelado e formação de tecnólogos.

É sabido que uma das condições para a realização da pesquisa documental é a averiguação da autenticidade dos documentos a serem analisados. No presente caso, a autenticidade é dada pela coleta dos dados diretamente do sítio eletrônico das próprias IES em que funcionam os respectivos cursos.

Outro aspecto a ser considerado é com relação à natureza do texto do documento. Cellard (2008) alerta sobre a necessidade de, antes de tirar conclusões, levar em conta a existência de diferentes estruturas nos textos dos documentos, que variam em razão de contextos. Muitas vezes são estruturados de forma a fazer sentido somente no contexto específico de sua produção. É precisamente o que ocorre no presente caso. 
Conforme já se apontou, os cursos superiores de graduação, no Brasil, têm como orientação principal as chamadas Diretrizes Curriculares, emanadas do Conselho Nacional de Educação. Em sendo assim, é lícito e adequado que tais diretrizes devem se consubstanciar no projeto pedagógico de cada curso.

No âmbito das instituições, o projeto pedagógico é o principal marco regulatório de cada um de seus cursos; reveste-se, então, de fundamental importância para as práticas universitárias, notadamente quanto a políticas institucionais de interdisciplinaridade. Devem conter a concepção de curso, o tipo de egresso que se espera formar, a estrutura didático-pedagógica, a matriz curricular, as relações ensino-pesquisa-extensão, a proposição da interdisciplinaridade e os processos de avaliação.

\subsection{Análise de Dados}

$\mathrm{Na}$ averiguação das propostas de interdisciplinaridade, as categorias foram formuladas, inicialmente, a partir das questões postas para o estudo. Porém, as categorias podem ser flexibilizadas uma vez que a integração é tida como aspecto essencial na busca da interdisciplinaridade no ensino superior (Klein, 2012).

Também as análises devem refletir preocupações no sentido de: (i) reconhecer a grande multiplicidade e variedade de perspectivas conceituais e ideias práticas no ensino superior; (ii) reconhecer que a construção de sentido holístico pode requerer uma prática em várias etapas; (iii) reconhecer que interdisciplinaridade constitui-se de relações entre disciplinas que continuam existindo.

Sob essas perspectivas, a proposta de categorias e análise de conteúdo dos projetos pedagógicos ficou assim formulada:

Tabela 1. Categorias de análise dos projetos pedagógicos de cursos

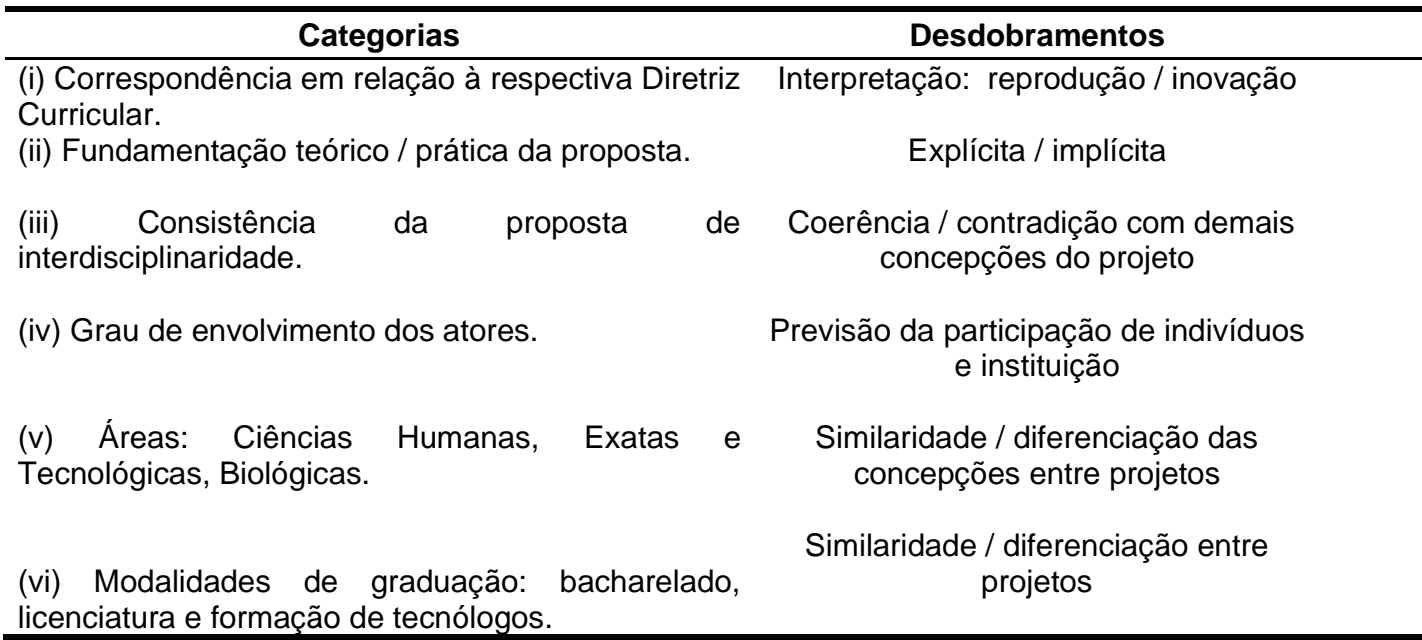

Procurar-se-á - como procedimento de análise temática - interpretar todas as variações semânticas, tendo em vista a integração e o contexto em que ocorrem.

\section{Conclusões}

Não se pode esperar que os resultados, em vista de coleta de dados ainda em fase inicial, sejam já esclarecedores. Portanto, como resultados, só podemos indicar que estão restritos à construção de um referencial teórico e ao instrumental para a análise dos dados. 
Segundo nos parece, com o trabalho até agora realizado, os resultados deverão indicar tanto aspectos facilitadores, quanto entraves à consecução de ações interdisciplinares nas IES. Temerário seria pressupor contribuição efetiva no âmbito produção filosófica ou metodológica.

Entretanto, acredita-se que o caminho interpretativo do método hermenêutico poderá conduzir aos desígnios de construção de saber significativo acerca do percurso entre a formulação de políticas públicas (de que fazem parte as Diretrizes Curriculares) e a sua prática efetiva no contexto acadêmico.

\section{Referências}

Associação Brasileira de Normas Técnicas. (2018). NBR 6023: informação e documentação referências - elaboração. ABNT.

Barreto, F. C., Marcelino, C. D. M., \& Olário, P. S. (2016). Interdisciplinaridade no ensino superior: reflexões e descrição de uma prática real. FCD.

Bassarab Nicolescu (2010). Para uma educação e aprendizagem transdisciplinares. In A. Z. Bassit (Org). O interdisciplinar: olhares contemporâneos (pp. 11-23). Factash.

Bortoni-Ricardo, S. M. (2013). O professor pesquisador: introdução à pesquisa qualitativa. Parábola.

Cellard, A. (2008). A análise documental. In J. Poupart, J. Deslauriers, L. Groulx, A. Laperrière, R. Mayer, \& A. P. Pires. A pesquisa qualitativa: enfoques epistemológicos e metodológicos (pp. 295-316). Vozes.

Coimbra, J. A. A. (2000). Considerações sobre a interdisciplinaridade. In A. Philippi Jr., C. E. M. Tucci, D. J. Hogan, \& R. Navegantes (Eds). Interdisciplinaridade em ciências ambientais (pp. 52-67). Signus.

Falcus S., Cameron C., Halsall JP (2019). Interdisciplinarity in higher education: the challenges of adaptability. Em: Snowden M., Halsall J. (Eds). In Mentorship, Leadership, and Research. Perspectivas internacionais sobre política, administração e prática social. Springer, Cham. https://doi.org/10.1007/978-3-319-95447-9_10

Fazenda, I. C. A. (2012). Interdisciplinaridade: história, teoria e pesquisa. 18ª ed. Papirus.

Fazenda, I. C. A., Tavares, D. E., \& Godoy, H. P. (2015). Interdisciplinaridade na pesquisa científica. Papirus.

Feldman, M. G. (2014). Formação docente e contexto institucional. In I. C. A. Fazenda, \& H. P. Godoy (Orgs). Interdisciplinaridade: pensar, pesquisar e intervir (pp. 52-63). Cortez.

Flick, U. (2009). Introdução à pesquisa qualitativa. (3ª ed.). Artmed.

Jantsch, A. P., \& Bianchetti, L. (Orgs) (2011). Interdisciplinaridade: para além da filosofia do sujeito. Vozes.

Japiassu, H. (1992). A atitude interdisciplinar no sistema de ensino. Revista Tempo Brasileiro. Rio de Janeiro, n. 108 (pp. 83-94), jan-mar.

Klein, J. T. (2012). Ensino interdisciplinar: didática e teoria. In I. C. A. Fazenda (Org). Didática e interdisciplinaridade, $17^{\mathrm{a}}$ ed. (pp. 109-132), Papirus.

Neves, F. (2019). Transdisciplinaridade: significados. https://www.significados.com.br/transdisciplinaridade.

Pena-Vega, A. (2010). Da interdisciplinaridade à complexidade na pesquisa. Conferência proferida no IV RECOPI, CAPES/MEC, Brasília-DF.

Roggero, R. (2010). O interdisciplinar na universidade. In A. Z. Bassit (Org). O interdisciplinar: olhares contemporâneos (pp. 139-152). Factash.

Santomé, J. T. (1998). Globalização e interdisciplinaridade: o currículo integrado. Tradução Cláudia Schilling. Artes Médicas. 
Vol. 7 | Investigação Qualitativa em Educação: Avanços e Desafios

Santos, M. N. dos, Rosa, C. T. W. da, \& Darroz, L. M. (2019). Interdisciplinaridade no contexto escolar: relato de uma atividade envolvendo um estudo sobre diferentes países. In Vivências, 15(28), 135-144. https://doi.org/10.31512/vivencias.v15i28.21

Silva, D. R. (2019). Currículos inovadores no ensino superior. Senac. 